\title{
»dare intellectum salutis populi eius ...« (Lc 1,77) Der Codex Bezae und der lateinische Text der lukanischen Cantica
}

Par

\section{Thomas Johann Bauer}

Universität Erfurt

$\mathrm{D}$ er in der Universitätsbibliothek Cambridge verwahrte Codex Bezae (Cantabrigiensis) ist eine griechisch-lateinische Bilingue, die mit einigen Lücken den Text der vier kanonischen Evangelien und der Apostelgeschichte sowie einen kleinen Abschnitt aus dem Dritten Johannesbrief überliefert. ${ }^{1}$ Die erhaltenen, zum Teil beschädigten 406 Blätter haben das Format 260 mal $220 \mathrm{~mm}$ und sind einspaltig mit 33 Zeilen beschrieben, wobei auf der linken Seite der griechische Text und auf der rechten Seite Zeile für Zeile die entsprechende lateinische Übersetzung notiert ist.

\section{Der Codex Bezae und der lateinische Bibeltext}

Die Evangelien stehen in der im Westen bzw. in der lateinischen Bibel zunächst vorherrschenden Ordnung (Mt, Jo, Lc, Mc). Der griechische Text der Evangelien und der Apostelgeschichte zeigt auffällige Abweichungen vom Text jener Papyri und Majuskelhandschriften,

\footnotetext{
${ }^{1}$ Codex Bezae (VL 5/d für den lateinischen Text; D/05 für den griechischen Text): Cambridge, University Library, Nn. II. 41. Vgl. AlAnd 1989, S. 118f.; GrYSON 1999, Bd. 1, S. 25f.; Fischer 1972, S. 39-43; Houghton 2016, S. 27f. u. 52; HAElewYCK 2013-18, S. 10f.; weiterführend PARKER, AMPHOUX (Hg.) 1996; PARKER 1992.
} 
die heute als die besten Zeugen des ältesten griechischen Textes gelten, und damit auch vom Text der maßgebenden kritischen modernen Editionen des griechischen Neuen Testaments. ${ }^{2}$ Etliche der Auffälligkeiten des griechischen Textes, darunter größere und kleiner Erweiterungen sowie Auslassungen, finden sich nicht in anderen Handschriften oder allenfalls in sehr wenigen Zeugen des neutestamentlichen Textes.

Der lateinische Text des Codex Bezae ist keine Neuschöpfung, wie deutliche Übereinstimmungen bzw. Berührungen mit den erhaltenen Zeugen der sogenannten Vetus Latina (Versio) also des älteren lateinischen Bibeltextes aus der Zeit vor Hieronymus (gest. 420) und der Vulgata zeigen. ${ }^{3}$ Zugleich aber nimmt der Codex Bezae auch unter den Zeugen des älteren lateinischen Texts der Evangelien und der Apostelgeschichte eine Sonderstellung ein, weil er sich immer wieder deutlich von den anderen Zeugen der älteren lateinischen Übersetzung unterscheidet. Deshalb wird in der Forschung vermutet, dass der übernommene lateinische Text bearbeitet wurde, um ihn besser dem benutzten griechischen Text anzupassen. Manche meinen außerdem, einige der Eigenheiten des griechischen Textes gingen darauf zurück, dass teilweise der griechische Text anhand des lateinischen Textes überarbeitet wurde. Damit lassen sich aber keineswegs alle Besonderheiten im griechischen Text des Codex Bezae erklären.

Der im Codex Bezae benutze lateinische Text scheint in seiner Herkunft nicht einheitlich zu sein, da er in den einzelnen Evangelien sowie der Apostelgeschichte unterschiedlichen Zeugen und Ausprägungen des älteren lateinischen Bibeltextes nahesteht. ${ }^{4}$ Im Matthäusevangelium zeigt sich öfter eine Nähe zum Codex Bobiensis (4. Jh.; Nordafrika) ${ }^{5}$ und zum Codex Palatinus (5. Jh.; wohl Trient) ${ }^{6}$ und damit zu einem Text der offenbar bereits im 3. Jahrhundert in Nordafrika verbreitet war. Im Lukasevangelium und im Johannesevangelium steht der lateinische Text des Codex Bezae zwischen dem Codex Palatinus und dem Codex Vercellensis (4. Jh.; wohl Vercelli) ${ }^{7}$ und nähert sich damit einer Form des Textes, wie sie im frühen 4. Jahrhundert in Italien belegt ist. Beim Markusevangelium zeigt sich eine noch größere Nähe zum Codex Vercellenis, öfter aber auch zu Zeugen einer Form des Textes, wie sie nach Mitte des 4. Jahrhunderts in Italien in Gebrauch war. In der Apostelgeschichte zeigt der lateinische Text des Codex Bezae eine große Nähe zu dem Text, wie er im 4. Jahrhundert

\footnotetext{
${ }^{2} \mathrm{Zu}$ besten Zeugen des ältesten griechischen Textes des Neuen Testaments rechnet man: Codex Sinaiticus (※/01; London, British Library, Add. 43725; 4. Jh.); Codex Alexandrinus (A/02; London, British Library, MS Royal 1 D. VIII; 5. Jh.); Codex Vaticanus (B/03; Città del Vaticano, Bibliotheca Apostolica Vaticana, Gr. 1209; 4. Jh.). Zu den Handschriften vgl. Aland 1989, S. 117f. Alter und Vorrang ihrer Textform bestätigt vor allem der Papyrus Bodmer XIV. XV ('P35; Città del Vaticano, Bibliotheca Apostolica Vaticana, Bodmer; 2./3. Jh.). Maßgebend zum textgeschichtlichen und textkritischen Wert von $\mathfrak{P}^{75}$ MARTINI 1966.

${ }^{3}$ Näheres zum lateinischen Text des Codex Bezae in Relation zu den Handschriften der Vetus Latina vgl. GRYSON 1999, Bd. 1, S. 26; für das Markusevangelium auch HAELEWYCK 2013-18, S. 73-78. 111.

${ }^{4} \mathrm{Zu}$ den Ausprägungen des Textes der älteren lateinischen Übersetzung, ihren Zeugen und Besonderheiten vgl. Fischer 1972, S. 24-39; Metzger 1977, S. 326-330; Burton 2000, S. 14-28; außerdem zu den methodischen Grundlagen FisCHER 1972, S. 6-18; VOGELS 1928a, S. 123-129; BURKITT 1920, S. 25-41.

${ }^{5}$ Codex Bobiensis (VL 1/b): Turin, Biblioteca Nazionale Universitaria, 1163 (G.VII.15). Vgl. FisCHER 1972, S. 30-32; Houghton 2016, S. 210; Gryson 1999, Bd. 1, S. 19f.; zum Text der Handschrift und seiner Einordnung vgl. auch BURTON 2000, S. 16-19.

${ }^{6}$ Codex Palatinus (VL 2/e): Trient, Museo Nazionale (Castello del Buon Consiglio s.n.); Dublin, Trinity College 1709 (N.IV.18); London, British Library, Add. 40107; vgl. GrYSON 1999, Bd. 1, S. 21f.; HoughtON 2016, S. $210 \mathrm{f}$.

${ }^{7}$ Codex Vercellensis (VL 3/a): Vercelli, Archivio Capitolare Eusebiano, s.n.; vgl. GRYsON 1999, Bd. 1, S. 23; Houghton 2016, S. 211.
} 
bei Lucifer von Cagliari (gest. 371) und deutlich später noch durch den Gigas librorum (frühes 13. Jh.; Böhmen) ${ }^{8}$ bezeugt ist.

Da sich aus dem griechischen und lateinischen Text sowie aus paläographischen und anderen Beobachtungen am Codex selbst keine eindeutigen und belastbaren Indizien ergeben, bleibt die Frage der Entstehung und Herkunft des Codex Bezae in der Forschung ebenso umstritten wie die Erklärung der Eigenheiten seines griechischen und lateinischen Textes. Der Codex gelangte 1562 in den Besitz des protestantischen Gelehrten Theodore Bezae, der ihn 1581 an die Universität in Cambridge schenkte. Angeblich stammte der Codex aus dem Kloster des Heiligen Irenaeus in Lyon, wo er im 8. Jahrhundert auch bezeugt ist und offenbar repariert wurde. Woher die Handschrift ursprünglich stammte und wie sie nach Lyon gelangte, ist unklar. Der in vielerlei Hinsicht singuläre Charakter des griechischen und lateinischen Textes lassen vermuten, dass er abseits der Zentren der Entstehung der großen und dominierenden Handschriften des griechischen Neuen Testaments entstanden ist. Als Ort, an dem der Codex geschrieben wurde, wird deshalb vielfach Beirut vermutet, teilweise jedoch auch Ägypten, Nordafrika, Gallien oder Italien. Die Entstehung wird meist um 400 angesetzt. Nach der Fertigstellung des Codex wurde der lateinischen Text an einigen Stellen überarbeitet und Inhaltsangaben, liturgische Vermerke, die eusebianischen Sektionszahlen und andere Ergänzungen nachgetragen.

\section{Der lateinische Text der Evangelien von Cyprian bis Hieronymus}

Darüber, wann, wo und wie die Übertragung der Bibel ins Lateinische begann, sind keine Nachrichten überliefert. ${ }^{9}$ Immerhin lassen die zahlreichen Schriftzitaten bei Cyprian von Karthago (gest. 258) erkennen, dass bereits vor Mitte des 3. Jahrhunderts in Nordafrika Übersetzungen für (fast) alle Bücher der Bibel vorlagen. ${ }^{10}$ Möglicherweise liegen die Anfänge des lateinischen Bibeltextes in den lateinisch-sprachigen christlichen Gemeinden Nordafrikas, in denen - anders als in Rom - immer weniger Mitglieder in der Lage waren, den griechischen Text der biblischen Schriften zu verstehen, so dass man wohl seit der Wende vom 2. zum 3. Jahrhundert bei gottesdienstlichen Versammlungen und in der Katechese zunehmend dazu überging, Schrifttexte ad hoc für die Anwesenden ins Lateinische zu übersetzen. ${ }^{11}$ Aus diesen zunächst mündlichen Übertragungen entwickelten sich wohl allmählich Übersetzungskonventionen, die dann sukzessive verschriftlicht wurden. Am Anfang dieses Prozesses der Übertragung standen wohl die am häufigsten gebrauchten biblischen Texte, so dass man davon ausgehen muss, dass zuerst Übersetzungen für die Psalmen und Evangelien vorlagen. Der Prozess der Übertragung ins Lateinische und der Verschriftlichung der Übersetzungen

\footnotetext{
${ }^{8}$ Gigas librorum (VL 51/gig): Stockholm, Kungliga Biblioteket, A. 148. Vgl. GRYsOn 1999, Bd. 1, S. 79f.; FISCHER 1972, S. 28 u. 29f.; HOUGHTON 2016, S. 233.

${ }^{9}$ Belege, die in der Regel für die Existenz erster lateinischer Übersetzungen in christlichen Gemeinden in Nordafrika, Gallien und Rom bereits nach der Mitte des 2. Jahrhunderts genannt werden, sind nicht eindeutig und nicht belastbar: Erwähnung von libri et epistulae Pauli uiri iusti in der Passio der Märtyrer von Scili (180 n.Chr. in Karthago); als Rückübersetzung aus dem Lateinischen interpretiertes Zitat von Eph 6,5 im griechisch verfassten Brief der Gemeinden von Lugdunum und Vienna (177 n.Chr.). Vgl. Houghton 2016, S. 5f. Für die Geschichte der lateinischen Bibel und ihres Textes im Überblick BAUER 2020, S. 17-56. Außerdem BARTELINK 2012, S. 2355-2362; Bogaert 1992, S. 799-803; Burton 2014, S. 167-200; Elliott 1992, S. 198-245; GRIBOMONT 1985, S. 43-65; KEDAR 1988, S. 299-338.

${ }^{10}$ Vgl. Houghton 2016, S. 9f.; außerdem Bauer 2020, S. $23-25$.

${ }^{11}$ Überlegungen zu den Anfängen und zur möglichen frühen Entwicklung der Übertragung der biblischen Schriften ins Lateinische bei BAUER 2020, S. 25f.
} 
wurde nicht »offiziell« initiiert und gesteuert, sondern verdankte sich der Initiative einzelner Gemeinden oder Personen. Deshalb konnte jeder, wenn er es für geboten hielt, den Text nach eigenem Belieben bearbeiten, um ihn stilistisch oder mit Blick auf den Text der griechischen Vorlagen zu verbessern. Dies dokumentieren bei fast allen biblischen Schriften zahlreiche Abweichungen in den erhaltenen Zeugnissen der älteren lateinischen Übersetzung aus der Zeit vor Hieronymus und der Vulgata.

Die auffällige Mischung aus Gemeinsamkeiten und Unterschieden in den verschiedenen Zeugnissen des älteren lateinischen Textes der einzelnen biblischen Bücher führte in der Forschung zu einer bis heute andauernden kontroversen Diskussion darüber, ob bei jedem biblischem Buch am Anfang überhaupt eine einzige Übersetzung steht, die sekundär bearbeitet und so unterschiedliche Ausprägungen gefunden hat, oder ob der Befund nicht eher dafür spreche, dass am Anfang mehrere unabhängige Übersetzungsversuche standen, die nachträglich einander beeinflusst haben und sich so erst sekundär einander angeglichen haben. ${ }^{12}$ Zurecht weist die neuere Forschung darauf hin, dass der Prozess für die einzelnen biblischen Bücher nicht einheitlich verlaufen sein muss, zumal die seltener gebrauchten Texte und Bücher wohl erst mit zeitlichem Abstand und im Kontext des Bemühens um die Herstellung einer vollständigen lateinischen Bibel übersetzt wurden.

Für die Evangelien votiert die Forschung heute mehrheitlich - wohl nicht zu Unrecht dafür, dass eine erste, aus mündlichen Vorformen hervorgegangene Übersetzung sukzessive verändert wurde, indem man gezielt Wörter ersetzte und eine neue veränderte kirchliche und theologische Begrifflichkeit eintrug. ${ }^{13}$ Außerdem war man offenbar zunehmend an einer möglichst wörtlichen Übertragung interessiert, so dass man als zu frei und paraphrasierend empfundene Übersetzungen korrigierte. Bei dem Bemühen um eine möglichst genaue Anpassung der lateinischen Übersetzung an die griechischen Vorlagen kam es auch zu Veränderungen, weil sich offenbar allmählich im Westen die Form des griechischen Textes durchsetzte, wie sie in den wichtigsten griechischen Bibelhandschriften des 4. Jahrhunderts bezeugt ist, und dabei eine abweichende Form des griechischen Textes verdrängte, wie sie im 2. und 3. Jahrhundert im Westen (aber auch in Syrien und an anderen Orten) verbreitet war. ${ }^{14}$ Aus einer veränderten Begrifflichkeit, aus der Anwendung divergierender Übersetzungstechniken und durch die Benutzung anderer griechischer Vorlagen entstehen abweichende Ausprägungen des älteren lateinischen Textes der neutestamentlichen Evangelien, die oft nebeneinander in Gebrauch bleiben und miteinander konkurrieren, worauf unter anderem Augustinus von Hippo (gest. 430) in einer vielzitierten Klage verweist (vgl. AU do 2,1617). ${ }^{15}$ Eine solche Ausprägung des lateinischen Textes ist auch die von Hieronymus - nach

\footnotetext{
${ }^{12}$ Knappe Anmerkungen zur Diskussion bei BuRTON 2000, S. 29f.; außerdem HougHTON 2016, S. 12-14, der für alle biblischen Bücher zur Annahme einer einzigen Übersetzung am Anfang tendiert.

${ }^{13}$ Für die charakteristischen »afrikanischen« Vokabeln der Vetus Latina vgl. BERGREN 1991, S. 175-205; vgl. auch FISCHER 1972, S. 8-14; außerdem VOGELS 1918, S. $251 \mathrm{ff}$.

${ }^{14}$ Die Vetus Latina (insbesondere ihre frühen Formen) werden mit griechischen Vorlagen verbunden, deren Text mit Antiochia und Syrien verbunden wird. Bei den neutestamentlichen Schriften nimmt man eine traditionell als »Westlicher Text« bezeichnete Form an, die man auch hinter den alten syrischen Übersetzungen (Vetus Syra) zu erkennen meint. Bei den neutestamentlichen Schriften hat diese Textform in der Überlieferung des griechischen Textes kaum Spuren hinterlassen. Zu Problem und Diskussion vgl. ALAND 1989, S. 57-81; für den »Westlichen Text« bes. 63f. Für die Vetus Syra zum Stand der Forschung WiLliams 2014, S. 143-166.

${ }^{15}$ Die Bewertung der Mehrgestaltigkeit des lateinischen Bibeltextes bei Augustinus und anderen Kirchenvätern ist ambivalent; je nach Situation kann als Bereicherung und Hindernis in theologischen Diskussionen gesehen werden. Vgl. dazu BAUER 2020, S. 29f.
} 
eigenen Angaben auf Veranlassung von Papst Damasus I. (reg. 366-384) ab 382 vorgenommene, oft wenig sorgfältige Revision der lateinischen Übersetzung der Evangelien, die später Teil der Vulgata wurde (die übrigen Schriften des Neuen Testaments, wie auch einige Schriften des Alten Testaments gehen nicht auf Hieronymus zurück). ${ }^{16}$

Beim Lukasevangelium, wie auch bei den anderen neutestamentlichen Evangelien, lassen sich für die Zeit vor der Revision durch Hieronymus anhand der vorhandenen Zeugen mehrere Ausprägungen des Textes (Texttypen) identifizieren: ${ }^{17}$ Ein ältere afrikanischer Texttyp, der den Zitaten bei Cyprian nahesteht, ist - wie bereits erwähnt - im Codex Palatinus (VL 2) sowie teilweise auch im Codex Colbertinus (VL 6; 12. Jh., Südfrankreich) ${ }^{18}$ erkennbar, auch wenn in beiden Handschriften der ältere afrikanische Text immer wieder durch Einflüsse eines jüngeren europäischen Textes überlagert wird, wie er etwa im Codex Corbeiensis (VL 8; 5. Jh., Italien) ${ }^{19}$ bezeugt ist. Der bereits genannte Codex Bobiensis (VL 1), der den älteren afrikanischen Text offenbar am besten überliefert, fällt aufgrund seiner fragmentarischen Erhaltung für das Lukasevangelium vollständig aus. Mit dem Codex Corbeiensis bilden der Codex Veronensis (VL 4; 5. Jh., wohl Verona ${ }^{20}$ und der fragmentarisch erhaltene Codex Vindobonensis (VL 17; 5. Jh., Italien) ${ }^{21}$ die Kerngruppe des europäischen Textes, wie er in Italien um 350-380 vorherrschte und Hieronymus als Grundlage für die Revision des Textes der Evangelien diente. ${ }^{22}$ Der Text dieser Handschriften steht den Zitaten bei Ambrosius (gest. 397) und Lucifer von Cagliari sowie dem Ambrosiaster (um 366-384) nahe. Eine ältere Form des europäischen Textes bezeugt der bereits genannte Codex Vercellensis (VL 3), dessen Text eine gewisse Verwandtschaft mit den Zitaten bei Novatian (gest. 258) zeigt und weitgehend mit dem Zitat von Lc 15,11-32 in einem Brief des Hieronymus an Papst Damasus I. übereinstimmt (HI ep 21,4).

Weitere Handschriften, die zu den Zeugen des älteren lateinischen Textes der Evangelien gerechnet werden, überliefern entweder eine Form des Textes, die jünger ist als der lateinische Text im Codex Bezae oder gehören zur Gruppe jener Handschriften, in denen die ältere Form des lateinischen Textes weitgehend vom Text der Vulgata überlagert wird. Wie bereits angemerkt, steht nach dem Urteil der Forschung der lateinische Text des Codex Bezae im Lukasevangelium zwischen dem Codex Palatinus und dem Codex Vercellenis und damit zwischen dem älteren afrikanischen und der einer frühen Form des europäischen Textes und

\footnotetext{
${ }^{16}$ Der später als Vulgata bezeichnete Bibeltext wurde wohl Mitte des 5. Jahrhunderts zusammengestellt und besteht aus Übersetzungen unterschiedlicher Herkunft, geht also nur zum Teil auf Hieronymus zurück (Evangelien, Bücher der hebräischen Bibel, einschließlich der griechischen Zusätze zu Daniel und Ester, sowie Tobit und Judit). Vgl. BAUER 2020, S. 31f.

${ }^{17}$ Die Gruppierung der Handschriften und ihre Einordnung in die Entwicklung der lateinischen Übersetzung der Evangelien folgt FISCHER 1972, S. 30-39; dazu bereits oben in Anm. 4; vgl. auch die dort genannte Literatur.

${ }^{18}$ Codex Colbertinus (VL 6/c): Paris, Bibliothèque nationale, lat. 254; vgl. GRYSON 1999, Bd. 1, S 27; Houghton 2016, S. 213; Burton 2000, S. 27.

${ }^{19}$ Codex Corbeiensis (VL 8/ff²): Paris, Bibliothèque nationale, lat. 17225; vgl. GRYSON 1999, Bd. 1, S 31; Houghton 2016, S. 214f.; BuRTON 2000, S. 20.

${ }^{20}$ Codex Veronensis (VL 4/b): Verona, Biblioteca Capitolare, VI (6); vgl. GRYsON 1999, Bd. 1, S 24; Houghton 2016, S. 212; Burton 2000, S. $19 f$.

${ }^{21}$ Codex Vindobonensis (VL 17/i): Neapel, Biblioteca Nazionale, lat. 3; vgl. GRYsON 1999, Bd. 1, S 40; Houghton 2016, S. 219; Burton 2000, S. 20.

${ }^{22} \mathrm{Zu}$ den Vorlagen des Hieronymus für seine Revision der lateinischen Übersetzung der Evangelien vgl. VoGELS 1928b. Eine kritische Bewertung dazu bei FISCHER 1972, S. 61-64; außerdem MeTZGER 1977, S. 352354.
} 
repräsentiert damit eine zur Zeit seiner Entstehung altertümliche Form des lateinischen Textes. Was diese abstrakte Verhältnisbestimmung und Einordnung konkret bedeutet, soll exemplarisch durch die Analyse der drei lukanischen Cantica - Magnificat (Lc 1,46-55), Benedictus (Lc 1,67-78) und Nunc dimittis (Lc 2,28-32) - gezeigt werden. Dabei zeigt sich die Entwicklung des lateinischen Textes der Evangelien hin zur Vulgata und es wird ersichtlich, welche Überlegungen und Untersuchungen nötig sind, bevor für den Codex Bezae Wechselwirkungen zwischen dem griechischen und lateinischen Text bzw. Herkunft und Ursprung seines griechischen und lateinischen Textes diskutiert und behauptet werden können. ${ }^{23}$

\section{Der Codex Bezae und der ältere lateinische Text der lukanischen Cantica}

Durch einen Vergleich der genannten Handschriften mit dem Text der Stuttgarter Ausgabe der Vulgata $\left(\mathrm{Vg}^{\mathrm{S}}\right)^{24}$ zeigen sich bei den drei lukanischen Cantica sehr deutlich die Charakteristika der älteren lateinischen Übersetzung in ihrer frühen afrikanischen Ausprägung und in ihren jüngeren europäischen Fassungen und damit auch Art und Umfang der Bearbeitung der älteren Übersetzung(en) durch Hieronymus. ${ }^{25}$ Dabei treten auch die Eigenheiten des lateinischen Textes im Codex Bezae (VL 5) deutlich hervor. Da sich für diese Eigenheiten mehrmals Parallelen in den biblischen Zitaten bei den lateinischen Kirchenvätern und älteren Kirchenschriftstellern finden, ist zu vermuten, dass einige von ihnen der benutzten Vorlage angehörten und nicht erst bei der Zusammenstellung des Codex Bezae durch Angleichung an seinen griechischen Text entstanden sind. ${ }^{26}$

Für den Vergleich wird zunächst der Text der Revision des Hieronymus nach der Stuttgarter Vulgata $\left(\mathrm{Vg}^{\mathrm{S}}\right)$ angegeben; darunter werden die Abweichungen in den Handschriften der älteren lateinischen Übersetzung (Vetus Latina) notiert. ${ }^{27}$ Der Codex Vindobonensis (VL 17) fällt aufgrund seiner fragmentarischen Erhaltung bei den lukanischen Cantica als Textzeuge vollständig aus. Der Codex Vercellensis (VL 3) hat aufgrund von Beschädigungen im Text von Magnificat und Benedictus zahlreiche Lücken. Soweit möglich und begründbar, wird der Text ergänzt; Lücken und Ergänzungen sind jedoch (soweit nötig) markiert. Auf vorhandene Korrekturen in den Handschriften wird durch ${ }^{\mathrm{K}}$, auf unkorrigierte Lesarten durch * verwiesen. An Abkürzungen wird lac. für Fehlstellen, om. für Auslassungen, corr. für vorgeschlagene Korrekturen und + für Erweiterungen im Text der Handschrift

\footnotetext{
${ }^{23}$ Auf eine Bewertung und Einordnung der sprachlichen Eigenheiten des lateinischen Textes im Codex Bezae (Vokabular, Syntax, Stil) wird im Folgenden verzichtet. Dazu Stone 1946; eine berechtigte Kritik dazu jedoch bei FISCHER 1972, S. 41, Anm. 131.

${ }^{24}$ Biblia Sacra iuxta vulgatam versionem (GRYSON 2007b). Ein partiell abweichender Text bei WORDSWORTH, WHITE, 1889-1954. Zu den Abweichungen der Stuttgarter Vulgata von der älteren Ausgabe von Wordsworth-White und zu ihren Ursachen vgl. die kurzen Angaben bei FISCHER 1972, S. 50-52; außerdem FISCHER 1955, S. 178-196.

${ }^{25} \mathrm{Zu}$ den Zeugnissen des frühen afrikanischen Textes des Neuen Testamentes mit einem Versuch der Rekonstruktion des Textes VON SODEN 1909. Zu den Vorlagen des Hieronymus mit Rekonstruktion des von ihm benutzten Textes vgl. VogELS 1928b. Eine kritische Bewertung dazu bei FISCHER 1972, S. 61-64.

26 Parallelen in den altkirchlich-patristischen Schriften entstammen dem im Vetus Latina-Institut gesammelten Material (online verfügbar als Vetus Latina Database unter http://www.brepolis.net). Für die Autoren und Schriften sowie für die im folgenden gebrauchten Sigla vgl. GRYSON 2007a. Hier auch die Angaben zu den maßgebenden bzw. vorhandenen Editionen.

${ }^{27}$ Der Text der Handschriften wurde anhand von im Vetus Latina-Institut Beuron vorhandener Photographien bzw. anhand digitaler Reproduktionen verglichen (soweit über die besitzenden Bibliotheken online verfügbar). Der Text der Handschriften ist auch zugänglich in JÜLICHER 1976.
} 
verwendet. Der griechische Text und seine Varianten sind dem Nestle-Aland (28. Aufl.) entnommen, Nachweise dort. ${ }^{28}$

a. MagnifiCAt (Lc 1,46-55) $\left[\mathrm{Vg}^{\mathrm{S}}\right]$ : 46 et ait Maria magnificat anima mea Dominum 47 et exultauit spiritus meus in Deo salutari meo $\mathbf{4 8}$ quia respexit humilitatem ancillae suae ecce enim ex hoc beatam me dicent omnes generationes $\mathbf{4 9}$ quia fecit mihi magna qui potens est et sanctum nomen eius $\mathbf{5 0}$ et misericordia eius in progenies et progenies timentibus eum $\mathbf{5 1}$ fecit potentiam in brachio suo dispersit superbos mente cordis sui $\mathbf{5 2}$ deposuit potentes de sede et exaltauit humiles $\mathbf{5 3}$ esurientes impleuit bonis et diuites dimisit inanes $\mathbf{5 4}$ suscepit Israhel puerum suum memorari misericordiae $\mathbf{5 5}$ sicut locutus est ad patres nostros Abraham et semini eius in saecula

46 ait] dixit 2. 5; Maria] Elisabeth 3 (4) - 47 exultauit] laetatus est $2 \mid$ in] super $2 \mid$ Deo] d(eo) $3 \mid$ salutari] salutare 2, (sa)lutare 3, saluatori 5 - $\mathbf{4 8}$ quia] (qui)a 3, quoniam 5, qui 6 | respexit] inspexit 2 | humilitatem] humi(li)tatem 3, dominus super humilitatem $5 \mid$ ancillae] anci(la)e $3 \mid$ ecce ... generationes] lac. 3 | enim] + enim 2 | ex hoc] amodo $5 \mid$ beatam] beata 2; dicent] dicunt 2 | generationes] nationes 2 - 49 quia ... qui] lac. 2 | quia] quoniam $2.5 \mid$ magna] magnalia $2,+$ dominus 5 | qui potens est] ille potens $2 \cdot \mathbf{5 0}$ misericordia] misericordiam $8^{*} \mid$ in ... progenies] in saecula 2, in saecula saeculorum saecula 3 , in saecula saeculorum 4. 6, in generationes et generationes 5 , in progeniem et progeniem 8 |timentibus eum] eis qui eum metunt 2 - $\mathbf{5 1}$ potentiam] fortitudinem 2, potestatem 3, uirtutem $5,+$ suam $6 \mid$ dispersit] disparsit 4. 5, dissipauit $3 \mid$ mente cordis sui] sensu corda illorum 2, cogitatione cordis eorum 5 , mente et corda eorum 3 , mente cordis ipsorum 4, $8^{*} \cdot \mathbf{5 2}$ desposuit] destruxit $2 \mid$ de sede] a sedibus $2.5 \mid$ et exaltauit humiles $\mid$ (et e)xalta(uit humiles) 3 - 53 esurientes ... et] lac. 3 | impleuit] inpleuit 5. 8, repleuit 6 | bonis] bonorum 5 | diuites] locupletes 2 | dimisit] dismisit 5 - 54 suscepit] adsumpsit 2 , adiubauit 5 , et suscepit $6 \mid$ puerum suum] pueri sui $5 \mid$ memorari] commemoratus est 2 , memorare 5 , memoria 6 , re(cordatus est?) $3 \mid$ misericordiae] + suae 2 . 3. $8^{\mathrm{K}}$, misericordiam 5 - 55 sicut locutus est ad] (sicut 1)ocutus e(st ad) $3 \mid$ Abraham] Abrahae 2. 4 | saecula] aeternum 2.3.5

Im lateinischen Text Magnificat scheint Hieronymus lediglich an zwei oder drei Stellen in den Text der von ihm benutzte Vorlage ändernd eingegriffen $\mathrm{zu}$ haben: in progenies et

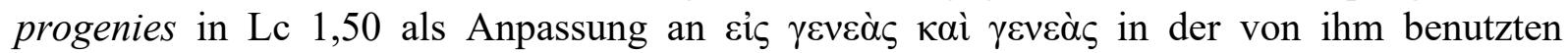
griechischen Vorlage; bei mente cordis sui in Lc 1,51 Ersatz von ipsorum oder eorum durch

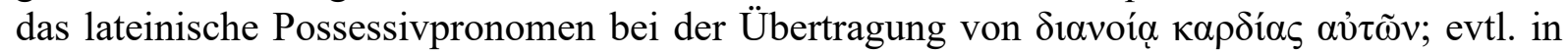
Lc 1,51 auch der Ersatz des stilistisch auffälligen Perfekt disparsit (bzw. dispcersit) durch das »reguläre« dispersit bei der Übersetzung von $\delta \imath \varepsilon \sigma \kappa o ́ \rho \pi \imath \sigma \varepsilon v .{ }^{29}$ Alles übrige entspricht dem Text wie er in Italien nach Mitte des 4. Jahrhunderts verbreitet war und in den Handschriften der europäischen Kerngruppe bezeugt ist (VL 4, 8).

\footnotetext{
${ }^{28}$ Novum Testamentum Graece (NeSTLE, AlAND 2012); außerdem The New Testament in Greek. The Gospel according to St. Luke (hrsg. von The American and British Committees of the International Greek New Testament Project), 2 Bde., Oxford, 1984/1987.

${ }^{29}$ Bezüglich des von Hieronymus für die Revision benutzten griechischen Evangelientextes merkt Houghton 2016, S. 33f., zurecht an: »It has often been observed that the Vulgate moves away from the socalled 'Western' text towards a form closer to the later standard (koine or Byzantine text), although attempts at more detailed classification tend to founder. In practice, Greek witnesses used by Jerome and other revisers would each have had their own peculiarities."
} 
Davon hebt sich im Codex Palatinus (VL 2) deutlich der ältere afrikanische Text mit zahlreichen Abweichungen ab: dixit statt ait in der Einleitungsformel in Lc 1,46; das freiere laetatus est statt exulatauit als Übersetzung für $\eta \gamma \gamma \alpha \lambda \lambda \dot{i} \alpha \sigma \varepsilon v$ und die wörtliche Übertragung von غ̇ंì $\tau \tilde{\omega}$ $\theta \varepsilon \tilde{\omega}$ mit super deo statt in deo in Lc 1,74; inspexit statt des präziseren respexit als Übertragung von $\dot{\varepsilon} \pi \varepsilon ́ \beta \lambda \varepsilon \psi \varepsilon v$, die Wiedergabe von $\mu \alpha \kappa \alpha \rho ı \tilde{\sigma} \sigma i ́ v \mu \varepsilon$ im Präsens mit beatam me dicunt statt im Futur und die ein leicht abweichendes Textverständnis andeutende Übersetzung von $\gamma \varepsilon v \varepsilon \alpha i$ mit nationes statt generationes in Lc 1,48; das für die alte afrikanische Übersetzung auch sonst charakteristische quoniam statt quia als Wiedergabe von ö $\tau$, das möglicherweise auf einem abweichenden griechischen Text ( $\mu \varepsilon \gamma \alpha \lambda \varepsilon \varepsilon \tilde{\alpha} \alpha$, auch im korrigierten Text des Codex Bezae) beruhende magnalia statt magna und das um eine

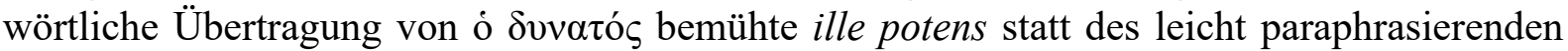
qui potens est in Lc 1,49; das mit keiner der Varianten des griechischen Textes übereinstimmende kurze in saecula statt in saecula saeculorum bzw. in progeniem et progeniem im

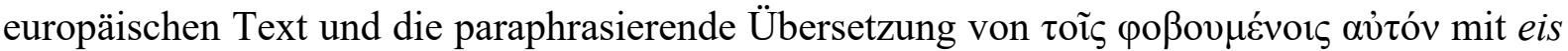
qui eum metunt anstelle der wörtlichen Übertragung timentibus eum in Lc 1,50; fortitudo statt

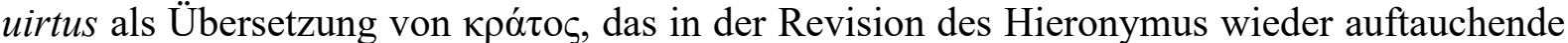

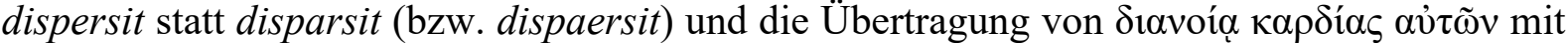
sensu corda illorum statt des semantisch und syntaktisch treffenderen mente cordis ipsorum (oder sui) in Lc 1,51; die Wiedergabe von $\kappa \alpha \theta \varepsilon \tilde{\imath} \lambda \varepsilon v$ mit destruxit statt mit dem im Blick auf den Kontext treffenderen deposuit und die Bewahrung des Plurals bei der Übersetzung von àmò $\theta \rho o ́ v \omega v$ mit a sedibus statt a sede in Lc 1,52; locupletes statt diuites als Übersetzung für

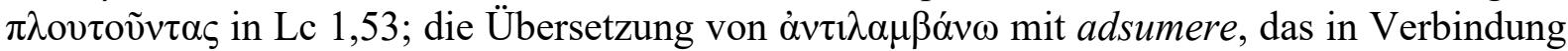
mit puer eher auf die Vorstellung der Adoption zielt, statt mit suscipere, das auf die Anerkennung eines Kindes als legitim zielt, sowie das syntaktisch vom griechischen Text abweichende commemoratus est statt memorari als Übertragung von $\mu \nu \eta \sigma \theta \tilde{\eta} v \alpha \iota$ und die ebenfalls vom griechischen Text abweichende präzisierende Ergänzung des Personal-

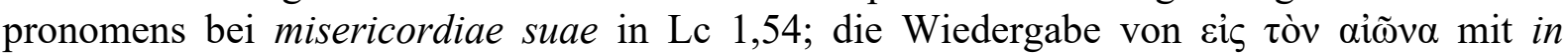
aeternum statt des späteren in saecula in Lc 1,55.

Auch wenn bei Cypian von Karthago, dem frühesten sicheren Zeugen für den ältesten lateinische Bibeltext kein Zitat aus dem Magnificat überliefert ist, zeigen Parallelen in Zitaten in der sonstigen altkirchlichen Literatur, dass der Codex Palatinus (VL 2) eine Form des lateinischen Textes des Lukasevangeliums bewahrt, die bereits vor, aber auch noch parallel zu dem von Hieronymus bei seiner Revision benutzten europäischen Text in Umlauf war. Das laetatus est in Lk 1,47 kennt auch noch Ambrosius (AM Ps 47,11,18). Hieronymus zitiert Lc 1,49 einmal ebenfalls mit quoniam (HI Is 3). Bei Hieronymus und in einer anonymen, um die Mitte des 4. Jahrhunderts in Nordafrika wohl im Kreis der Donatisten entstandenen Sammlung von Prophetien findet sich ein Zitat von Lc 1,51 mit fortitudo (AN pro pg. 20; HI Lc 8). Diese Sammlung bestätigt für Lc 1,51 die Formulierung sensu corda illorum, für Lc 1,52 destruxit und a sedibus und für Lc 1,53 locupletes (vgl. dazu ebenfalls AN pro pg. 20). Die wahrscheinlich noch im 3. Jahrhundert entstandene alte lateinische Übersetzung der Schrift Adversus haereses des Irenaeus von Lyon, die in den biblischen Zitaten oft mit Cyprian übereinstimmt, zitiert Lc 1,54 mit adsumpsit (bzw. assumpsit) und misericordiae suae (IR 3,10,2). Auch Augustinus belegt das Possessivpronomen suae (AUG Ev 2,17). Die lateinische Übersetzung des Irenaeus zitiert außerdem Lc 1,55 mit in aeternum (IR 3,10,2).

Der Codex Vercellensis (VL 3) stimmt - wie auch der Codex Corbeiensis (VL 6) - im Magnificat weitgehend mit dem Text der Handschriften der europäischen Kerngruppe überein. Dazu gehört auch in saecula saeculorum (saecula) in Lc 1,50 als Wiedergabe der

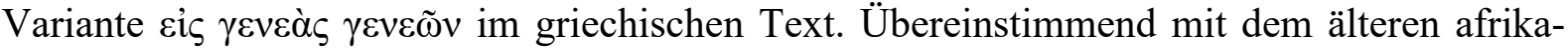
nischen Text bewahrt er jedoch in Lc 1,55 in aeternum anstelle des europäischen in saecula. 
Eigenheiten des Codex Vercellensis sind potestas statt potentia, dissipavit statt disparsit und mente et corda eorum in Lc 1,51 sowie recordare oder reminsci statt memorari in Lc 1,54.

Der Codex Bezae (VL 5) hat im Vergleich dazu im Magnificat mehr Elemente des afrikanischen Textes bewahrt: dixit in der Einleitungsformel in Lc 1,46; quoniam in Lc 1,49; der Plural a sedibus in Lc 1,52; in aternum in Lc 1,55. Nähe zum afrikanischen Text zeigt auch quoniam in Lc 1,48 (hier quia jedoch in VL 2). Einige Eigenheiten des Codex Bezae haben Parallelen in Zitaten aus dem Magnifikat in der altkirchlichen Literatur und könnten einer älteren Form der lateinischen Übersetzung angehören. Die auffällige wortgetreue

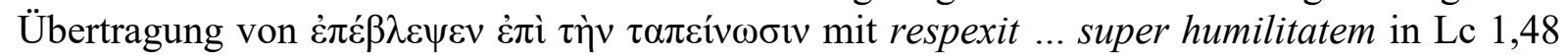
überliefert auch Hieronymus (HI Lc 8), ebenso die Übersetzung von ảंò $\tau$ oṽ vṽv mit amodo im selben Vers (HI Is 3; Lc 8). Singulär ist jedoch in Lc 1,47 saluatori statt salutari (salutare) und in Lc 1,48 dominus nach respexit (und entsprechend кúptos im griechischen Text). Singulär ist ebenfalls deus (und entsprechend ó $\theta \varepsilon o ́ s$ ) als Bezugswort vor qui potens est in Lc

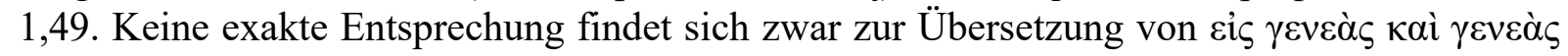
mit in generationes et generationes in Lc 1,50; doch bezeugt Hieronymus für Lc 1,50 mit in generationes generationum bzw. a generatione in generationem zumindest dieselbe Wort-

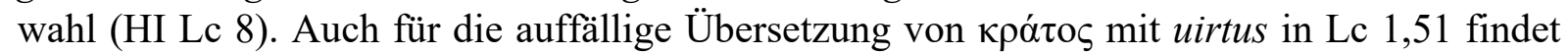
sich eine Parallele bei Hieronymus (HI Lc 8). Keine Parallele hat allerdings cogitatione cordis eorum im selben Vers. Ebenfalls singulär ist in Lc 1,53 der offensichtlich um eine wörtliche Übertragung des griechischen Textes bemühte Genitiv in inpleuit bonorum und in Lc 1,54 die sehr freie Wiedergabe von $\alpha v \tau \imath \lambda \alpha \mu \beta \alpha ́ v \omega$ mit adiuuare mit dem folgenden, syntaktisch nicht passenden Genitiv pueri sui als genaue Entsprechung zum griechischen Text. Singulär ist ebenfalls der Akkusativ misericordiam in Lc 1,54, eine Angleichung an den griechischen Text ist wohl das Fehlen des Possessivpronomens (so auch im europäischen Text).

b. Benedictus (Lc 1,67-78) [ $\left.\mathrm{Vg}^{\mathrm{S}}\right]$ : 67 et Zaccharias pater eius impletus est Spiritu Sancto et prophetauit dicens $\mathbf{6 8}$ benedictus Deus Israhel quia uisitauit et fecit redemptionem plebi suae 69 et erexit cornu salutis nobis in domo Dauid pueri sui 70 sicut locutus est per os sanctorum qui a saeculo sunt prophetarum eius $\mathbf{7 1}$ salutem ex inimicis nostris et de manu omnium qui oderunt nos $\mathbf{7 2}$ ad faciendam misericordiam cum patribus nostris et memorari testamenti sui sancti $\mathbf{7 3}$ iusiurandum quod iurauit ad Abraham patrem nostrum $\mathbf{7 4}$ daturum se nobis ut sine timore de manu inimicorum nostrorum liberati seruiamus illi $\mathbf{7 5}$ in sanctitate et iustitia coram ipso omnibus diebus nostris $\mathbf{7 6}$ et tu puer propheta Altissimi uocaberis praeibis enim ante faciem Domini parare uias eius 77 ad dandam scientiam salutis plebi eius in remissionem peccatorum eorum $\mathbf{7 8}$ per uiscera misericordiae Dei nostri in quibus uisitauit nos oriens ex alto 79 inluminare his qui in tenebris et in umbra mortis sedent ad dirigendos pedes nostros in uiam pacis

67 et Zaccharias] et Zac(charias) 3 | pater eius] (p)ater eius 3, om. 4 | impletus] inpletus 5, repletus 8 | spiritu sancto] sancto spiritu $2 \mid$ prophetauit] $\mathrm{p}$ (ro)phetauit 3, prophetabat 2. 4. 6, om. $5 \mid$ dicens] (di)cens 3, dixit 5 - 68 deus] dominus deus 2.5 | quia] qui 2 | uisitauit] prospexit 2 | et fecit] om. 2 | redemptionem] salutem 5 | plebi suae] populo suo 2. 5, populo 3 , plebis suae 6.4 .8 • 69 erexit] excitauit 2, elebauit (corr. eleuauit) 5, lac. in $3 \mid$ cornu] cornum 2. 5. $8^{*}$, corn(..) $3 \mid$ salutis] lac. 3 | domo] (dom)o 3, domum 6 | Dauid pueri sui] Da(...) 3 - 70 sicut] lac. 3 | locutus] (locu)tus 3, corr. loc(ut)us $2 \mid$ per ... eius] per os sanctorum suorum prophetarum qui a principio temporis sunt 2 , per os sanctorum prophetarum suorum qui a principio sunt 6 , per os sanctorum prophetarum eius qui a saeculo 5, per os sanctorum p(rop)h(etarum) su(oru)m qui (a) saeculo sunt 3, per os sanctorum prophetarum qui ab aeuo sunt 4.8 - 71 salutem] et liberauit nos 4. 8. 6, lac. 3 | ex] ab 2. 4. 6. 8, lac. 3 | inimicis] (ini)micis $3 \mid$ manu] ma(..) 3, corr. man(ib)us $2 \mid$ ex ... omnium] de manu inimicorum 
nostrorum et omnium $5 \mid$ qui oderunt nos] qui nos oderunt 2. (3.) 4. 6 - 72 ad faciendam] facere 2. 5 . $3 \mid$ misericordiam] + suam $8^{*} \mid$ cum] om. $8^{*} \mid$ patribus $\mid$ nostris] n(os)tris $3 \mid$ memorari] commemorari 2, (...)ra(...) 3, memoratus est 6 , rememorari $8 \mid$ t. sui sancti] t. sancti sui 2.4. 6. 8, t. sancti eius 5, lac. 3 - 73 iusiurandum] om. 2, iuramentum 5, iusiurandi 8, lac. $3 \mid$ quod iurauit] lac. $3 \mid$ ad ... nostrum] abrahae patri nostro $2,(\ldots)$ trum 3 - $\mathbf{7 4}$ daturum se nobis] ut det nobis 2 , ut daret nobis 5 , daturum nobis se nobis $8^{*} \mid$ ut] om. 2.5 | timore] metu $2 \mid$ de manu] om. 2 | nostrorum] om. 2, liberati] om. 2, tos 5. 3 | seruiamus] seruire 2.3. 5 | illi] ipsi 3, ei 5. 6 - 75 sanctitate] ueritate 2, castitate 3 | iustitia] iusti(tia) $3 \mid$ coram ipso] in conspectu eius 2. (3.) $5 \mid$ omnibus ... nostris] omnes dies nostros (3.) 5 • $76 \mathrm{tu}]+$ autem $5 \mid$ puer] infans 5 | propheta altissimi] (pro)pheta Alt(is)simi 3 | uocaberis] uocaueris 2. 4. $5\left(8^{*}\right) \mid$ praeibis] antecedes $5 \mid($ an)te facie $(\mathrm{m}) 3 \mid$ parare] praeparare $2.6, \mathrm{p}(\ldots) 3 \mid$ uias] uiam 8 - 77 ad dandam] ad mandandam 2, (...)dandam 3, dare $5 \mid$ scientiam] agnitionem 2, (sci)entiam 3, intellectum 5; plebis eius] populo suo 2, populo eius 3, populi eius $5 \mid$ remissionem] remissa 2, remissione $5 \mid$ eorum] suorum 2, ipsorum $3 \cdot 78$ per] propter $2.5 \mid$ misericordiae] (mi)sericordiae $2 \cdot$ 79 inluminare] praelucere $2,+$ lumen 5 , illuminare $6.8^{\mathrm{K}} \mid$ his] eis 2 , iis (3.) $4 \mid$ tenebris] + sunt $2.6 \mid$ in] om. 5 , qui in $8 \mid$ sedent] sedentibus 2. 5.6, sedentes 3 , sedet $8^{*} \mid$ ad dirigendos] ut prosperefaciat $5 \mid$ in] ad 2

Auch am lateinischen Text des Benedictus, wie ihn die Hauptzeugen des europäischen Textes (VL 4, 8) dokumentieren, hat Hieronymus bei seiner Revision kaum Änderungen vorgenommen: in Lc 1,68 Ersatz des Genitiv plebis suae durch den Dativ plebi suae zur Angleichung an den griechischen Text; in Lc 1,69 vielleicht Änderung des selteneren cornum zu cornu (falls er dies nicht bereits in der Vorlage fand; vgl. VL 4. $8^{\mathrm{K}}$ ); in Lc 1,70 sanctorum qui a saeculo sunt prophetarum eius statt sanctorum prophetarum qui ab aeuo (oder saeculo) sunt, offensichtlich zur Angleichung an eine veränderte griechische Vorlage (wohl $\tau \tilde{\omega} v \dot{\alpha} \gamma i ́ \omega v$

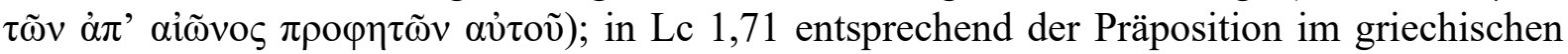
Text ex inimicis nostris anstelle von ab inimicis nostris; in Lc 1,72 gegen die griechische Wortstellung testamenti sui sancti statt testamenti sancti sui, wohl um den Bezug des Possessivpronomens und sancti als zugehöriges Adjektiv deutlicher zu machen.

Demgegenüber zeigt auch beim Benedictus der Codex Palatinus (VL 2) einige deutliche Abweichungen: in Lc 1,67 das Imperfekt prophetabat statt des Perfekt prophetauit; ebenfalls

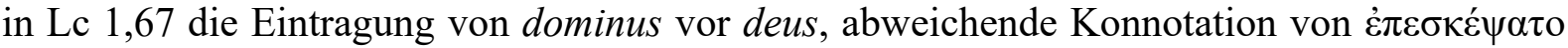
durch die Wiedergabe mit prospexit statt uisauit und damit verbunden das Übergehen von кaì

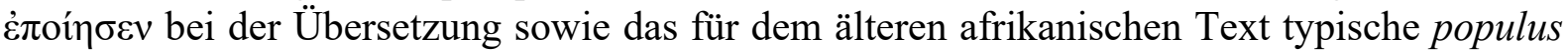
anstelle von plebs als Übersetzung für $\lambda \alpha$ ós; in Lc 1,69 excitauit statt erexit als Wiedergabe

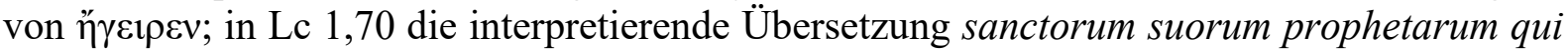

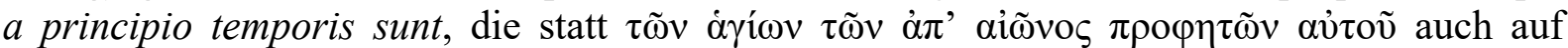

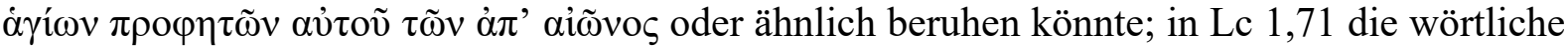
Wiedergabe von $\sigma \omega \tau \eta \rho i ́ \alpha v$ mit salutem statt der paraphrasierenden und glättenden Übertragung mit et liberauit sowie manibus als vermutliche Korrektur zu manus in der Handschrift; in Lc 1,72 der dem griechischen Text entsprechende einfache Infinitiv facere statt ad faciendam und commemorari statt (re-)memorari in den jüngeren Formen des Textes; in Lc 1,73 das Übergehen von óркоv bei der Übersetzung und die Wiedergabe von $\pi \rho \grave{\varsigma} \varsigma$ A $\beta \rho \alpha \alpha ̀ \mu \kappa \tau \lambda$. mit dem Dativ Abrahae etc. statt der wortgetreuen Übertragung ad Abraham

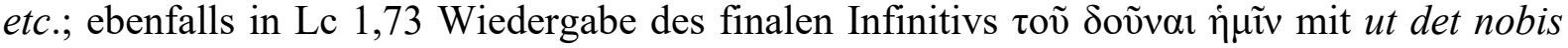
statt daturum se nobis; in Lc 1,74 Fortführung mit Infinitiv-Konstruktion entsprechend dem griechischen Text statt Auflösung in $u t$-Satz; dabei sine metu statt sine timore für $\alpha \dot{\varphi} \rho ́ \beta \omega \varsigma$ und

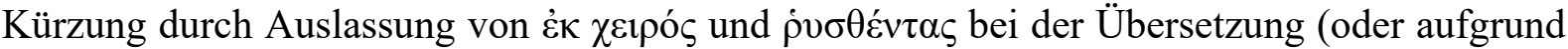
einer abweichenden griechischen Vorlage); in Lc 1,74 die auffällige Übersetzung von ó $\sigma$ ió mit ueritas statt sanctitas und in conspectu statt coram als Wiedergabe von Évó 


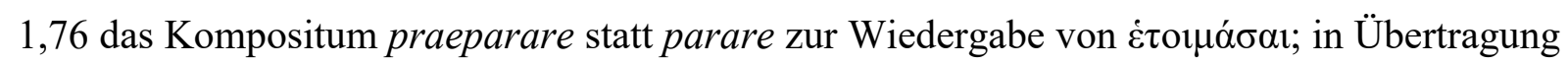

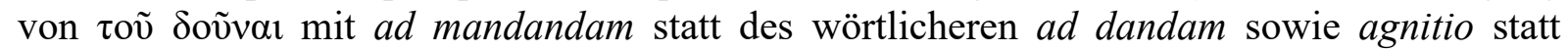
scientia als Übersetzung von $\gamma v \tilde{\sigma} \sigma 1 \varsigma$, das ungewöhnliche remissa statt remissio zur Wiedergabe von ă $\varphi \varepsilon \sigma 1 \varsigma$ und nach peccatorum das Possessivpronomen suorum statt des wörtlicheren eorum; in Lc 1,78 das interpretierende propter uiscera statt des mehr wörtlicheren per uiscera für $\delta i \alpha ̀ ~ \sigma \pi \lambda \alpha ́ \gamma \chi v \alpha$; in Lc 1,79 offensichtlich im Bemühen um eine sehr wörtliche Übertragung

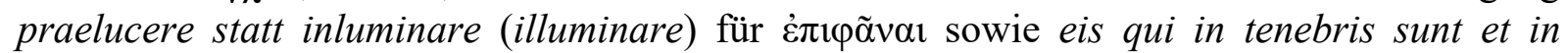
umbra mortis sedentibus statt der glättenden Wiedergabe his qui in tenebris et in umbra

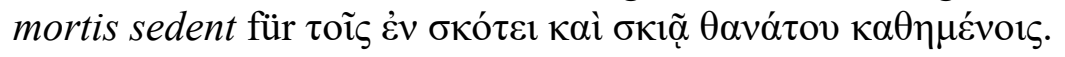

Parallelen in Zitaten aus dem Benedictus in der altkirchlichen Literatur, darunter auch Cyprian von Karthago, zeigen, dass der Text des Codex Palatinus (VL 2) zwar die ältere afrikanischen Form der Übersetzung weitgehend gut überliefert, dass aber nicht alle seine Eigenheiten dieser älteren Form des lateinischen Textes zugerechnet werden können. Ein Zitat bei Cyprian bestätigt das Imperfekt prophetabat in der Einleitungsformel Lc 1,67 als alten afrikanischen Text (CY te 2,7); es findet sich unter anderem auch noch bei Ambrosius und in der schon genannten anonymen donatischen Sammlung von Prophetien (AM Lc 2,33; AN pro pg. 55.31). Anders als der Codex Palatinus zitiert Cyprian, wie auch die anonyme donatistische Sammlung von Prophetien, Lc 1,68 entsprechend der Mehrheit der lateinischen Überlieferung ohne dominus vor deus Israhel (CY te 2,7; AN pro pg. 21), so dass dominus wohl nicht zum alten afrikanischen Text zu rechnen ist, sondern als spätere Ergänzung unter dem Einfluss der dominierenden Form des griechischen Textes zu werten ist. Die Ergänzung dominus findet sich aber in der alten lateinischen Übersetzung des Irenaeus, so dass sie zumindest Teil einer älteren Textform sein dürfte (HI Lc 10; IR 3,10,1). Für Lc 1,68 bezeugt Cyprian auch das typisch afrikanische populus für $\lambda \alpha$ ó $\varsigma$ und er zitiert den Vers ebenfalls in

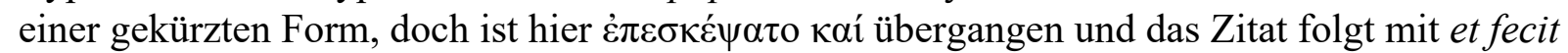
redemptionem dem üblichen lateinischen Text (CY te 2,7 [vl prospexit]). Die anonyme donatistische Sammlung von Prophetien zitiert Lc 1,68 mit aspexit redemptionem und könnte ein Hinweis sein, dass der Codex Palatinus (VL 2) hier doch eine in Nordafrika vor Mitte des 4. Jahrhunderts verbreiteten Form des Textes bewahrt hat (AN pro pg. 21 u. 55 [vl prospexit]). Die anonyme donatistische Sammlung von Prophetien bezeugt zudem in Lc 1,69 excitauit statt prospexit (AN pro pg. 21); Cyprian dagegen zitiert mit suscitauit, das sich auch bei Hieronymus und später findet (CY te 2,7; HI Is 2; Lc 10). Für das auffällige sanctorum suorum prophetarum qui a principio temporis sunt in Lc 1,70 finden sich bei den Zitaten in den altkirchlichen Schriften keine Parallelen (am nächsten VL 6). Das facere am Anfang von Lc 1,72 belegt auch Hieronymus (HI Lc 10), doch fehlen Hinweise, dass es zum älteren afrikanischen Text gehört; die alte lateinische Übersetzung des Irenaeus hat das dominierende ad faciendam (IR 3,10,3). Auch für commemorari im selben Vers fehlen Belege, dass es Teil

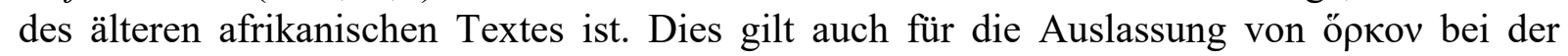
Übersetzung und den Dativ Abrahae patri nostro in Lc 1,73. Das ut det nobis am Ende desselben Verses bietet dagegen auch die alte lateinische Übersetzung des Irenaeus (IR 3,10,3). Auch für die stark abgekürzte Fassung des lateinischen Textes von Lc 1,74 fehlen Parallelen in den Zitaten. Auch sine metu und liberari sind in den Zitaten nicht belegt (sine metu allenfalls indirekt in HI Lc 10). Für in conspectu eius in Lc 1,75 weist die alte lateinische Übersetzung des Irenaeus auf Zugehörigkeit zum älteren afrikanischen Text. Für praeparare in Lc 1,76 belegt eine Parallele bei Hieronymus, dass es zu einer älteren Form des Textes gehört, und Parallelen bei Augustinus und Quodvultdeus (gest. um 453) weisen nach Nordafrika (HI Lc 10; AU Ev 2,17; QU Jud 14,6; pro 2,25). Bei Lc 1,77 fehlen Parallelen für ad mandandam und remissa, doch findet sich neben dem für den älteren afrikanischen Text charakteristischen populus auch agnitio in der alten lateinischen Übersetzung des Irenaeus (IR 
3,10,3). Unsicher ist propter uiscera in Lc 1,78, das ein Zitat bei Prosper von Aquitanien (gest. 455) belegt (PROS voc 1,19). Für Lc 1,79 fehlen Hinweise für Alter und Herkunft des abweichenden Textes im Codex Palatinus.

Auch im Benedictus entspricht der Codex Vercellensis (VL 3) - wie der Codex Corbeiensis (VL 6) - sehr weitgehend dem Text der europäischen Hauptgruppe (VL 4, 8). Entsprechungen zum Codex Palatinus sind in Lc 1,72 das offensichtlich ältere facere statt ad faciendam, in Lc 1,74 seruire ipsi (ei in VL 2) satt seruiamus illi (oder ei), in Lc 1,75 in conspectu eius statt coram ipso und in Lc 1,77 populo eius (suo in VL 2). Der Infinitiv seruire in Lc 1,74 bedingt den Akkusativ liberatos statt liberati im Text der europäischen Kerngruppe (VL 4, 8; mit VL 6 und $\mathrm{Vg}^{\mathrm{S}}$ ). Nähe zum Codex Palatinus zeigt auch populo statt plebis suae in Lc 1,68. Gegen den Codex Palatinus und die europäische Kerngruppe (mit VL 6 und $\mathrm{Vg}^{\mathrm{S}}$ ) steht in Lc 1,75 der Akkusativ omnes dies nostros statt des Ablativs. Singulär sind in Lc 1,73

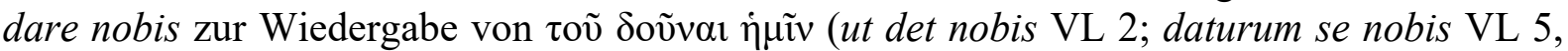
$6,8)$, in Lc 1,75 castitas als Wiedergabe für óøió $\rceil \varsigma$ und in Lc 1,79 das syntaktisch auffällige sedentes statt sedentibus im älteren afrikanischen Text bzw. sedent im jüngeren europäischen Text.

Der Codex Bezae (VL 5) zeigt auch im Benedictus im Vergleich zum Codex Vercellensis deutlich Übereinstimmung mit dem Codex Palatinus und dem älteren afrikanischen Text. Gemeinsam ist den drei Handschriften facere in Lc 1,72, seruire (ei) in Lc 1,74 und in conspectus eius in Lc 1,57. Außerdem haben alle drei Handschriften in Lc 1,77 das für den afrikanischen Text charakteristische populus, doch steht im Codex Bezae abweichend vom griechischen Text statt Dativ der Genitiv populi eius. Darüber hinaus finden sich im Codex Bezae als Gemeinsamkeiten mit dem Codex Palatinus dominus deus statt deus und populo suo statt plebis suae in Lc 1,68 (populo in VL 3), cornum statt cornu in Lc 1,69 (VL 3 hier beschädigt), propter uiscera statt per uiscera in Lc 1,78 und sedentibus statt sedent in Lc 1,79. Gegen den Codex Palatinus stimmt der Codex Bezae mit dem Codex Vercellensis lediglich in Lc 1,74 überein, wo der Infinitiv seruire den Akkusativ liberatos bedingt, sowie in Lc 1,75 bei omnes dies nostros. Von den Eigenheiten des Codex Bezae haben einige Parallelen in einer Form des Textes, die offenbar auch Hieronymus kennt (HI Lc 10): das auffällige de manu inimicorum nostrorum et omnium qui oderunt nos in Lc 1,71 (de manu omnium inimicorum nostrorum et de manu omnium qui oderunt nos); iuramentum statt iusrurandum und ut daret nobis in Lc 1,73 (vgl. ut det nobis in VL 2); antecedes statt paeibis in Lc 1,76. Die Wiedergabe von $\gamma v \tilde{\omega} \sigma 1 \varsigma$ mit intellectus in Lc 1,77 findet sich auch in der alten lateinischen Übersetzung des Irenaeus (IR 3,10,3). Ohne Parallelen sind: dixit statt prophetabat/prophetauit dicens in Lc 1,67; salutem statt redemptionem in Lc 1,68; eleuauit statt excitauit bzw. erexit in Lc 1,69; sanctorum profetarum eius qui a saeculo in Lc 1,70; et tu autem statt et tu und infans statt puer in Lc 1,76; dare statt ad (man)dandam und in remissione statt in remissionem/remissa in Lc 1,77; lumen nach inluminare und ut prosperefaciat statt ad dirigendos in Lc 1,79.

c. NUNC DIMITTIS (Lc 2,28-32) [VgS $]$ : 28 et ipse accepit eum in ulnas suas et benedixit Deum et dixit 29 nunc dimittis servum tuum Domine secundum verbum tuum in pace $\mathbf{3 0}$ quia viderunt oculi mei salutare tuum $\mathbf{3 1}$ quod parasti ante faciem omnium populorum $\mathbf{3 2}$ lumen ad revelationem gentium et gloriam plebis tuae Israhel

28 accepit] adcepit $8^{*} \mid$ in ulnas suas] in manus suas 2.6 , in amplexum 3 , in manibus 4 , in alas suas 5 , om. $8^{*} \mid$ dixit] locutus est 2 , ait 3 - 29 dimittis] dimitte 2.4 , dimittes 6 , dismittis 5 | seruum] serbum 5 - 30 quia] quoniam $6.8 \mid$ salutare] salutarem $8^{*}$ - 31 parasti] praeparasti $5 \mid$ ante faciem omnium] 
secundum omnium faciem 3 | in conspectu omnium 5 - 32 ad] in 2. 5, om. 3 | reuelationem] relatione 3 | gentium] oculorum 2, om. 5 | et] sed 2; gloriam] -a 6 | plebis tuae] populi tui 2. 3. 5, plebi 6

Beim Nunc dimittis hat Hieronymus in der Einleitungsformel in Lc 2,28 im Bemühen um

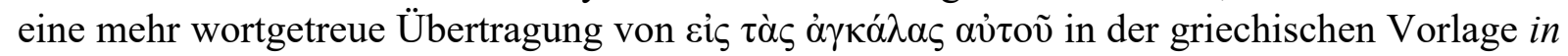
manibus durch in ulnas suas ersetzt. Im Text des Nunc dimittis selbst hat er, offensichtlich gegen den Text der griechischen Vorlage, lediglich in Lc 2,32 den Akkusativ gloriam durch den Nominativ gloria ersetzt und den Sinn des Textes verschoben. Christus ist so nicht mehr das lumen, das für die Völker reuelatio und für Israel gloria bedeutet, sondern Christus ist lumen für die Völker und gloria für Israel.

Deutlich weniger hebt sich im Nunc dimittis auch der Text des Codex Palatinus (VL 2) vom Text der Vorlage des Hieronymus ab: in Lc 1,28 in manus suas statt in manibus als

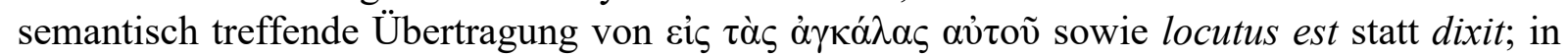
Lc 1,29 der Imperativ dimitte statt Indikativ dimittis (aber dimitte auch in VL 4); in Lc 1,32 die wörtliche Übertragung der Präposition ei` mit in statt des passenderen $a d$, das im Blick auf den griechischen Text irritierende occulorum statt gentium und das den Gegensatz zwischen den Völkern und Israel betonende sed statt et.

Zitate bei Cyprian und in der altkirchlichen Literatur zeigen zumindest die Nähe der Eigenheiten des Codex Palatinus zum älteren afrikanischen Text. Cyprian zitiert (paraphrasierend) Lc 2,28 mit in manus (CY mort 3); Belege bei Augustinus lassen jedoch vermuten, dass später in Nordafrika Lc 2,38 auch mit in manus suas verbreitet war (AU s 163,4; s Mor 3,6; vgl. Ps-AU s Cas I,131). Das dixit statt locutus est in Lc 2,28 bei Cyprian könnte der Tatsache geschuldet sein, dass ein freies Zitat vorliegt. Der Imperativ dimitte in Lc 2,29 findet sich zwar bei Ambrosius (AM Jos 9; Lc 2,57); Cyprian zitiert den Vers jedoch mehrfach mit dimittis (CY mort 3; te 3,58; vgl. PS-CY Vig 3). Die bereits mehrfach genannte anonyme donatistische Sammlung von Prophetien zitiert Lc 2,32 ebenfalls mit in reuelationem occulorum, so dass fraglich ist, ob diese auffällige Variante tatsächlich als Verschreibung von in reuelationem populorum zu erklären ist (AN pro pg. 21). Die alte lateinische Übersetzung von Irenaeus hat immerhin ad reuelationem occulorum (IR 4,7,1) neben in reuelationem gentium und belegt auch das für den älteren afrikanischen Text typische populi tui bei Israel (IR 3,10,5; 3,16,4). Keine Parallele hat jedoch das sed in Lc 2,32.

Am Anfang von Lc 2,30 hat der Codex Corbeiensis (VL 6) das in der älteren afrikanischen Übersetzung meist bevorzugte quoniam statt quia (VL 2), wie es auch Cyprian bezeugt (CY mort; PS-CY Vig 3, allerdings quia in CY te 3,58). Ansonsten bewahrt er in Lc 2,28 mit in manus suas die ältere afrikanische Form des Textes. Der Codex Vercellensis (VL 3) folgt auch beim Nunc dimittis weitgehend dem Text der europäischen Kerngruppe (VL 4, 8), zeigt aber einige Eigenheiten: in Lc 2,28 in amplexum statt in manibus bzw. in manus suas und ait statt dixit; in Lc 2,31 praeparasti statt parasti und das auffällige secundum omnium faciem populorum statt ante faciem omnium populorum; in Lc 2,32 relatione statt ad reuelationem (wohl infolge Textverderbnis).

Der Codex Bezae (VL 5) stimmt beim Nunc dimittis lediglich in Lc 2,32 bei in revelationem und gloriam populi tui Israhel mit dem im Codex Palatinus bezeugten älteren afrikanischen Text überein und wie der Codex Vercellensis hat er in Lc 2,31 praeparasti statt parasti (vgl. unter anderem auch IR 3,10,5; 3,16,4; 4,7,1). Die auch mit in conspectu singulär abweichende Formulierung des gesamten Verses hat unter anderem eine Parallele bei Hieronymus (HI Is 14). Singulär sind außerdem in Lc 2,28 in alas suas statt in manus suas bzw. in manibus, in Lc 2,29 dismittis statt dimittis (vgl. auch Lc 1,53) und in Lc 2,32 das Fehlen $\dot{\varepsilon} \theta v \tilde{\omega} v$ und einem lateinischen Äquivalent. 


\section{Anmerkungen zur Wertung des lateinischen Textes im Codex Bezae}

Im Magnifikat (Lc 1,46-55) und im Benedictus (Lc 1,67-78) steht der lateinische Text des Codex Bezae (VL 5) dem Text des Codex Vercellensis (VL 3) nicht wesentlich näher als den Handschriften der europäischen Hauptgruppe (VL 4, 8) oder auch der Revision des Hieronymus $\left(\mathrm{Vg}^{\mathrm{S}}\right){ }^{30}$ Dies ist der Tatsache geschuldet, dass Hieronymus sowohl im Magnificat als auch im Benedictus kaum Änderungen an dem von ihm benutzten lateinischen Text vorgenommen hat und dieser Text vermutlich eng mit dem der erhaltenen Zeugen der europäischen Hauptgruppe verwandt war. Deutlich erkennbar sind dagegen in beiden Cantica die Berührungen mit dem älteren afrikanischen Text, wie er für das Lukasevangelium am besten durch den Codex Palatinus (VL 2) bezeugt ist. Beim Nunc dimittis (Lc 2,28-32) verschiebt sich das Bild ein wenig, da der lateinische Text des Codex Bezae hier kaum Berührungen mit dem älteren afrikanischen Text hat, wie ihn der Codex Palatinus, ergänzt durch Belege in Zitaten in der altkirchlichen Literatur, belegt. Allerdings stimmt der lateinische Text des Codex Bezae beim Nunc dimittis auch nur einmal auffällig mit dem Codex Vercellensis überein, der sich hier jedoch stärker als bei den beiden anderen Cantica des Lukasevangeliums von den Zeugen der europäischen Hauptgruppe und damit auch von der Revision des Hieronymus unterscheiden lässt.

Mit Blick auf die lukanischen Cantica bestätigt sich demnach nicht das allgemeine Urteil der Forschung, der lateinische Text des Codex Bezae stehe im Lukasevangelium zwischen dem Codex Palatinus und dem Codex Vercellensis. Es ist demnach damit zu rechnen, dass die Nähe zu beiden Handschriften innerhalb des Lukasevangeliums von Abschnitt zu Abschnitt sehr stark variieren kann. Dass der Befund zur älteren lateinischen Übersetzung bei den lukanischen Cantica davon beeinflusst wäre, dass die Überlieferung ihres Textes sich aufgrund ihres liturgischen Gebrauchs vom Rest des Lukasevangeliums unterscheide, kann wohl ausgeschlossen werden; denn die lukanischen Cantica scheinen im Westen erst spät der Reihe der biblischen Cantica, die in der Liturgie verwendet wurden, hinzugefügt worden zu sein. ${ }^{31}$

An den lukanischen Cantica zeigt sich zudem, dass der lateinische Text des Codex Bezae keineswegs so singulär ist, wenn man beim Vergleich neben den Handschriften auch die Zitate in der altkirchlichen Literatur als Zeugnisse der älteren lateinischen Übersetzung aus der Zeit vor der Vulgata berücksichtigt. ${ }^{32}$ Auffällig sind insbesondere Parallelen bei Hieronymus in seiner Übersetzung der Homilien des Origenes zum Lukasevangelium (HI Lc). Es scheint nicht auszuschließen, dass der lateinische Text des Codex Bezae hier in höherem Maße, als man meist annimmt, einen im 4. Jahrhundert neben anderen gebräuchlichen Text repräsentiert. ${ }^{33}$ Man darf dabei zum einen nicht vergessen, dass die ältere lateinische Übersetzung kein einheitlicher Text war, sondern in verschiedenen Ausprägungen (Texttypen) in Umlauf war; zum anderen ist zu bedenken, dass die Zeugnisse dieser älteren

\footnotetext{
${ }^{30}$ Gegen das Urteil bei BURTON 2000, S. 23.

${ }^{31}$ Näheres zu Überlieferung und Gebrauch der lukanischen Cantica bei SCHNEIDER 1938.

32 Vgl. aber die Anmerkungen zu den mit biblischen Zitaten in der altkirchlich-patristischen Literatur verbundenen Fragen und Problemen bei JOSEF 1972, S. 455-478.

${ }^{33}$ Aufgrund des Befundes an den lukanischen Cantica scheint der lateinische Text des Codex Bezae jedenfalls ein differenzierteres und vorsichtigeres Urteil zu erfordern, als es z.B. ALAND 1989, S. 119, formuliert: »Der lateinische Text ist vom griechischen abhängig, er fällt aus der sonstigen altlateinischen Überlieferung heraus und stellt offensichtlich ein Sekundärprodukt dar.« Dies gilt auch für das Urteil bei BURTON 2000, S. 23: »The Latin translation is highly eccentric but is recognized as basically European with a strong African Element.«
} 
Übersetzung insgesamt gering sind und man deshalb nicht beurteilen kann, inwiefern das auf ihnen beruhende Bild repräsentativ und zutreffend ist. Was aufgrund der durch Zufall bestimmten Überlieferung als singulär erscheint, kann in wesentlich höherem Maße gebräuchlicher Text gewesen sein.

Mit Blick auf den Codex Bezae und seinem in der Forschung kontrovers diskutierten griechischen und lateinischen Text sollte man sich deshalb vor jedem pauschalen und vorschnellen Urteil hüten. Es scheint vielmehr geraten, Vers für Vers jede Besonderheit unter Einbeziehung des gesamten verfügbaren Materials zum griechischen und lateinischen Text der Evangelien und der Apostelgeschichte zu analysieren und $\mathrm{zu}$ evaluieren und so von Fall zu Fall zu festzustellen, ob Parallelen zu seinem griechischen und lateinischen Text vorliegen, und dann zu entscheiden, ob die Annahme einer Beeinflussung des lateinischen Textes durch den griechischen oder umgekehrt als Erklärung von Auffälligkeiten möglich und nötig ist. Erst auf diesem Weg wird sich im Durchgang durch die Handschrift ein genaueres Bild der Besonderheiten im griechischen und lateinischen Text des Codex Bezae ergeben, das ein verlässlicheres Urteil über die Herkunft und den Ursprung seines griechischen und lateinischen Textes ermöglicht.

Wechselwirkungen zwischen dem griechischen und lateinischen Text des Codex Bezae sind im Magnificat zu diskutieren für: in Lc 1,47 das singuläre $\dot{\varepsilon} v \tau \tilde{\varphi} \theta \varepsilon \tilde{\varphi}$ statt $\dot{\varepsilon} \pi \grave{i} \tau \tilde{\varphi} \theta \varepsilon \tilde{\omega}$ als Anpassung an das dominierende in deo der lateinischen Überlieferung; in Lc 1,48 dominus in der lateinischen Überlieferung offenbar ebenso isoliert wie кúpı $\varsigma$ in der griechischen; so auch in Lc 1,49 deus bzw. ó $\theta \varepsilon o ́ \varsigma$ (nicht verzeichnet als vl im Nestle-Aland). Beim Benedictus sind zu diskutieren: in Lc 1,67 die im griechischen und lateinischen Text singuläre kürzere

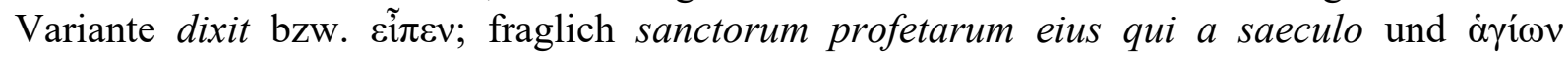

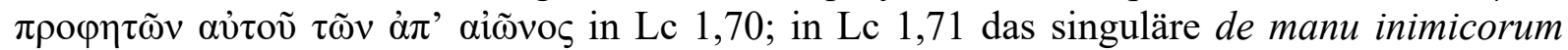

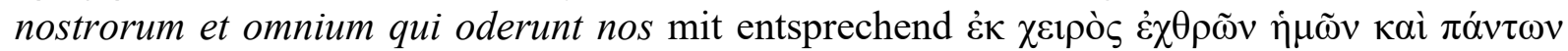
$\tau \tilde{\omega} \nu \mu 1 \sigma o v ́ v \tau \omega \nu \dot{\eta} \mu \tilde{\alpha} \varsigma$ (nicht verzeichnet als vl im Nestle-Aland); in Lc 1,79 das singuläre lumen und $\varphi \tilde{\omega} s$. Beim Nunc dimittis scheint lediglich das Fehlen von gentium und entsprechend $\dot{\varepsilon} \theta v \tilde{\omega} v$ in Lc 2,32 diskutabel. $\mathrm{Zu}$ ergänzen sind Fälle, bei denen klar eine Angleichung des lateinischen an den griechischen Text zu vermuten ist: in Lc 1,48 super humilitatem; in Lc 1,50 eventuell in generationes et generationes; in Lc 1,53 bonorum; in Lc 1,70 die Ergänzung von eius nach prophetarum; eventuell in Lc 1,75 omnes dies nostros; in Lc 1,76 autem nach et tu; in Lc 1,77 in remissione. 


\section{LITERATURVERZEICHNIS}

Aland, Kurt und Barbara, 1989 : Der Text des Neuen Testaments. Einführung in die wissenschaftichen Ausgaben und in die Theorie wie Praxis der modernen Textkritik, 2. Aufl., Stuttgart.

BARTELINK, Gerard J.M., 2012: »Frühe Bibelübersetzungen: von der Vetus Latina zur Vulgata des Hieronymus«, in Harald KITTEL u.a. (Hg.), Übersetzung - Translation Traduction. Ein internationales Handbuch zur Übersetzungsforschung, 3. Teilband, Berlin, Boston, S. 2355-2362.

BAUER, Thomas Johann, 2020 : »Von der Vetus Latina zur Nova Vulgata. Streiflichter zur Geschichte der lateinischen Bibel«, in Andreas Müller, Katharina HEYdEN (Hg.), Bibelübersetzungen in der Geschichte des Christentums. Theologische Anstöße (Veröffentlichungen der Wissenschaftlichen Gesellschaft für Theologie 62), Leipzig, S 17-56.

BERGREN, Theodore A., 1991 : A Latin-Greek Index of the Vulgate New Testament Based on Alfred Schmoller's Handkonkordanz zum griechischen Neuen Testament (Resources for Biblical Studies 26), Atlanta.

Bogaert, Pierre-Maurice, 1992 : »Latin Versions «, in David Noel Freedman (Hg.), The Anchor Bible Dictionary, Bd. 6, New York, S. 799-803.

BURKITT, Francis Crawford, 1920 : »Itala Problems«, in Scritti Varii Di Letteratura Ecclesiastica Dedicati Al Rev. Mo. Abate Ambrogio Amelli, O. S. B., Cassinese, in Occasione Del Cinquantesimo Anniversario Della Sua Ordinazione Sacerdotale, 1870-20 Settembre 1920, Monte Cassino, S. 25-41.

Burton, Philip, 2000 : The Old Latin Gospels. A Study of Their Texts and Language (Oxford Early Christian Studies), Oxford.

- 2014: »The Latin Versions of the New Testament«, in Bart D. EHRMAN, Michael W. Holmes (Hg.), The Text of the New Testament in Contemporary Research. Essays on the Status Quaesionis (New Testament Tools, Studies, and Documents 42), 2. Aufl., Leiden, S. 167-200.

ElliotT, J. Keith, 1992 : »Translations of the New Testament into Latin. The Old Latin and the Vulgate«, in Wolfgang HAASE, Hildegard TEMPORINI (Hg.), Aufstieg und Niedergang der Römischen Welt, Bd. II.26.1, Berlin, New York, S. 198-245.

FISCHER, Bonifatius, 1955 : »Der Vulgata-Text des Neuen Testaments«, Zeitschrift für neutestamentliche Wissenschaft 46, S. 178-196.

- 1972 : »Das Neue Testament in Lateinischer Sprache«, in Kurt AlAND (Hg.), Die alten Übersetzungen des Neuen Testaments, die Kirchenväterzitate und Lektionare (Arbeiten zur neutestamentlichen Textforschung 5), Berlin, New York, S. 1-92.

GRIBOMONT, Jean, 1985 : »Les plus anciennes traductions latines«, in Jacques FonTAINE, Charles Pietri (Hg.), Le monde latin antique et la Bible (Bible de tous les temps 2), Paris, S. 43-65.

GRYSON, Roger, 1999 : Altlateinische Handschriften / Manuscrits Vieux Latins. Repertoire descriptif, 2 Bde. (Vetus Latina 1,2A-B), Bd. 1, Freiburg im Breisgau.

- 2007a: Répertoire général des auteurs ecclésiastiques latins de l'antiquité et du haut moyen-âge, 2 Bde. (Vetus Latina 1,1), 5. Auflage, Freiburg im Breisgau. 
- 2007b : Biblia Sacra iuxta vulgatam versionem adiuvantibus B. Fischer, I. Gribomont, H.F.D. Sparks, W. Thiele, recensuit et brevi apparatu instruxit Robertus Weber, editionem quintam emendatam retractatam praeparavit Roger Gryson, Stuttgart.

HAELEWYCK, Jean-Claude, 2013-18: Evangelium secundum Marcum (Vetus Latina. Die Reste der altlateinischen Bibel 17), Freiburg/Br.

Houghton, Hugh A.G., 2016 : The Latin New Testament. A Guide to its Early History, Texts, and Manuscripts, Oxford.

JOSEF, Hermann, 1972: „Die Zitate des Neuen Testaments bei den lateinischen Kirchenvätern«, in Kurt AlAND (Hg.), Die alten Übersetzungen des Neuen Testaments, die Kirchenväterzitate und Lektionare (Arbeiten zur neutestamentlichen Textforschung 5), Berlin, New York, S. 455-478.

JÜLICHER, Adolf, 1976 : Itala. Das Neue Testament in altlateinischer Überlieferung, Bd. 3: Lucasevangelium (nach den Handschriften hrsg. von Adolf Jülicher, durchgesehen und zum Druck besorgt von Walter Matzkow und Kurt Aland), 2. verbesserte Auflage, Berlin, New York.

KedAR, Benjamin, 1988 : »The Latin Translations«, in Martin J. Mulder (Hg.), Mikra. Text, Translation, Reading and Interpretation of the Hebrew Bible in Ancient Judaism and Early Christianity (Compendia Rerum Iudaicarum ad Novum Testamentum 2,1), Assen, Maastricht, S. 299-338.

MARTINI, Carlo M., 1966 : Il problema della recensionalità del codice B alla luce del papiro Bodmer XIV (Analecta Biblica 26), Rom.

Metzger, Bruce M., 1977 : The Early Versions of the New Testament. Their Origin, Transmission and Limitations, Oxford.

Nestle, Eberhard u. Erwin, Aland, Kurt u. Barbara, 2012 : Novum Testamentum Græce (hrsg. vom Institut für Neutestamentliche Textforschung Münster/Westfalen unter der Leitung von Holger Strutwolf), 28. rev. Aufl., Stuttgart.

PARKer, David C., 1992 : Codex Bezae. An Early Christian Manuscript and Its Text, Cambridge.

PArker, David C., Amphoux, Christian-Bernard (Hg.), 1996 : Codex Bezae. Studies from the Lunel Colloquium, June 1994, Leiden.

SCHNEIDER, Heinrich, 1938 : Die altlateinischen biblischen Cantica (Texte und Arbeiten. 1. Abt.: Beiträge zur Ergründung des älteren lateinischen christlichen Schrifttums 29-30), Beuron.

Stone, Robert C., 1946 : The Language of the Latin Text of Codex Bezae. With an Index Verborum (Illinois Studies in Language and Literature 30, 2/3), Urbana.

The New Testament in Greek. The Gospel according to St. Luke (hrsg. von The American and British Committees of the International Greek New Testament Project), 2 Bde., Oxford, 1984/1987.

VoGELS, Heinrich Joseph, 1918 : »Zur >afrikanischen` Evangelienübersetzung«, in Biblische Zeitschrift 12, S. 251ff.

- 1928a: »Übersetzungsfarbe als Hilfsmittel zur Erforschung der neutestamentlichen Textgeschichte«, Revue Bénédictine 40, S. 123-129. 
- 1928b : Vulgatastudien. Die Evangelien der Vulgata untersucht auf ihre lateinische und griechische Vorlage (Neutestamentliche Abhandlungen 14, 2-3), Münster.

VON Soden, Hans, 1909 : Das Lateinische Neue Testament in Afrika zur Zeit Cyprians nach Bibelhandschriften und Väterzeugnissen, Leipzig.

Williams, Peter J., 2014 : »The Syriac Versions of the New Testament«, in Bart D. EHRMAN, Michael W. Holmes (Hg.), The Text of the New Testament in Contemporary Research. Essays on the Status Quaestionis (New Testament Tools, Studies, and Documents 42), 2. Aufl., Leiden, S. 143-166.

WordswOrTH, John, White, Henry Julian, u.a. (eds.), 1889-1954 : Nouum Testamentum Domini Nostri Iesu Christi Latine. Secundum Editionem Sancti Hieronymi, 3 Bde., Oxford. 


\title{
ZUSAMMENFASSUNG
}

Der zweisprachige Codex Bezae (Cantabrigiensis) ist eine der prominentesten Handschriften und ein wichtiger Zeuge des griechischen und lateinischen Textes der Evangelien und der Apostelgeschichte. Zahlreiche Besonderheiten und singuläre Lesarten lassen fragen, wie er in die Textgeschichte des griechischen und lateinischen Neuen Testaments eingeordnet werden kann. Am Beispiel der lukanischen Cantica lässt sich zeigen, dass erst eine möglichst vollständige Berücksichtigung des Materials ein differenziertes Urteil über Herkunft und Entstehung seines Textes erlaubt und tatsächliche Wechselwirkungen zwischen dem Text der benutzten griechischen und lateinischen Vorlagen identifizierbar macht.

\begin{abstract}
The bilingual Codex Bezae (Cantabrigiensis) is one of the most prominent manuscript and an important witness of the Greek and Latin text of the Gospels and Acts. Numerous peculiarities and singular readings raise question how it should be placed within the textual history of the Greek and Latin New Testament. Using the Lukan Canticles as an example, it can be shown that a well-founded knowlegde of the material preserved is required in order to obtain a more differentiated understanding of the origin of the Greek and Latin text of the codex and the actual relationship between both texts.
\end{abstract}

\section{SCHLÜSSELWÖRTER}

1.Lateinische Bibel

2. Textkritik

3.Lukasevangelium

4.Codex Bezae

5.Vetus Latina

\section{KEYWORDS}

1.Latin Bible

2.Textual Criticism

3. Gospel of Luke

4.Codex Bezae

5.Vetus Latina 